\title{
CRIAÇÃO E VALIDAÇÃO DE UMA BASE DE DADOS COM ELEMENTOS DO TRÂNSITO BRASILEIRO PARA VEÍCULOS AUTÔNOMOS
}

\author{
Diego Haji Carvalho Campos* Elder de Oliveira Rodrigues ** \\ Erick Carvalho Campos*** \\ * Departamento de Computação e Construção Civil, CEFET-MG Rua \\ 19 de Novembro, 121 - Centro Norte, Timóteo - MG, (e-mail: \\ diegohaji@gmail.com). \\ ** Departamento de Computação e Construção Civil, CEFET-MG Rua \\ 19 de Novembro, 121 - Centro Norte, Timóteo - MG (e-mail: \\ elderr@cefetmg.br) \\ *** Setor de Infraestrutura, UFJF-GV Rua São Paulo, 745, Centro, \\ Governador Valadares - MG (e-mail: erick.campos@ufjf.edu.br)
}

\begin{abstract}
Machine learning has enabled the emergence of revolutionary new products and resources, such as autonomous vehicles. Due to artificial neural networks applied to computer vision, systems have become able to recognize patterns approximately to human vision. For deep neural network training a large database is required to ensure visual patterns understanding. Aiming to contribute to the applications for autonomous vehicles, this work creates a database with some elements of Brazilian traffic signaling. For database training and validation, Yolov3 network was used and tests were performed in several scenarios, evaluating criteria such as the number of objects detected and the ability to correctly identify the type of object. As a result, for the virtual environment there was a detection of $21,5 \%$ of objects, of which $83 \%$ were correctly classified. And for the real scenario there was a detection of $61,8 \%$ of objects, which $88 \%$ of these were classified correctly.
\end{abstract}

Resumo: A aprendizagem de máquina vem possibilitando o surgimento de novos produtos e recursos revolucionários, como veículos autônomos. Graças às redes neurais artificiais aplicadas à visão computacional, os sistemas conseguem reconhecer padrões de forma próxima à visão humana. Para o treinamento de redes neurais profundas (Deep Learning) é necessária uma base de dados numerosa para a compreensão de padrões visuais. Visando contribuir com as aplicações para veículos autônomos, este trabalho cria uma base de dados com alguns elementos do trânsito brasileiro. Para a validação e treinamento da base de dados foi utilizada a rede Yolov3 e realizados testes em diversos cenários, avaliando critérios como a quantidade de objetos detectados e a capacidade de identificação correta do tipo de objeto. Como resultado, para o ambiente virtual houve detecções de $21,5 \%$ de objetos, com $83 \%$ classificados corretamente. E para os cenários reais houve detecções de $61,8 \%$ de objetos, com $88 \%$ classificados corretamente.

Keywords: Machine Learning; Autonomous Vehicle; Artificial Neural Network; Deep Learning; Yolo-v3; Database.

Palavras-chaves: Aprendizagem de Máquina; Veículos Autônomos; Redes Neurais Artificiais; Deep Learning; Yolo-v3; Base de Dados.

\section{INTRODUÇÃO}

Uma das tecnologias com maior destaque no ramo da computação contemporânea é Machine Learning (ML) ou aprendizado de máquina, que é um campo da inteligência artificial (IA) e fornece aos sistemas a capacidade de aprender e melhorar de forma autônoma a partir da experiência sem ser explicitamente programado. O cerne do campo, segundo Patterson and Gibson (2017) é a obtenção de descrições estruturadas a partir de dados brutos. De acordo com LeCun et al. (2015), a tecnologia de ML está inserida em muitos aspectos da sociedade moderna, como em motores de buscas na web, filtragem autônoma de conteúdo em redes sociais, nas recomendações em sites de comércio eletrônico, e está cada vez mais presente em produtos de consumo como câmeras e smartphones. Os sistemas de aprendizado de máquina são usados para identificar objetos em imagens em tempo real, interpretar linguagem falada e selecionar resultados relevantes da pesquisa de acordo com o contexto do usuário. Cada vez mais, essas aplicações fazem uso de um método de promoção de aprendizado de máquina denominada Deep Learning (DL). A 
figura 1, retirada de NVIDIA (2018b), apresenta de forma esquemática a relação entre IA, ML e DL.

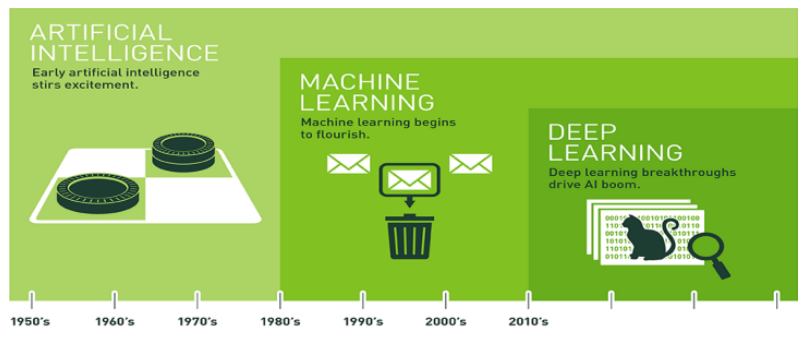

Figura 1. Diferenças entre IA,ML,DL.

A definição de deep learning de acordo com Patterson and Gibson (2017) é um tipo de rede neural com um grande número de parâmetros e camadas em uma das arquiteturas de redes neurais básicas (redes pré-treinadas não-supervisionadas, redes neurais convolucionais, redes neurais recorrentes, redes neurais recursivas). $\mathrm{O}$ aspecto principal da técnica de Deep learning é que essas camadas de recursos não são projetadas por humanos: elas são construídas a partir de dados usando um procedimento de aprendizado para fins gerais LeCun et al. (2015)

De acordo com Krizhevsky et al. (2012), as abordagens atuais para o reconhecimento de objetos em visão computacional (VC) fazem uso essencial dos métodos de aprendizado de máquina, contribuindo diretamente com a evolução dos veículos autônomos. Devido a natureza visual das informações de trânsito, como as sinalizações que determinam regras de circulação das vias, os sensores convencionais como o radar não são suficientes para viabilizar um veículo efetivamente autônomo, necessitando de reconhecimento visual do ambiente para tal.

O DL como técnica computacional tem contribuído para a ampliação da capacidade de reconhecimento de objetos complexos em veículos autônomos e melhoria do tempo de resposta dos algoritmos de inteligência artificial, pois quanto maior a base de referências disponível mais apto o veículo estará para circular num ambiente real cuja variabilidade é inerente e as ações precisam ser em tempo real. Um marco nesse campo foi o lançamento do Firefly em 2015 (Waymo, 2019), o primeiro carro autônomo do Google, como visto na figura 2.

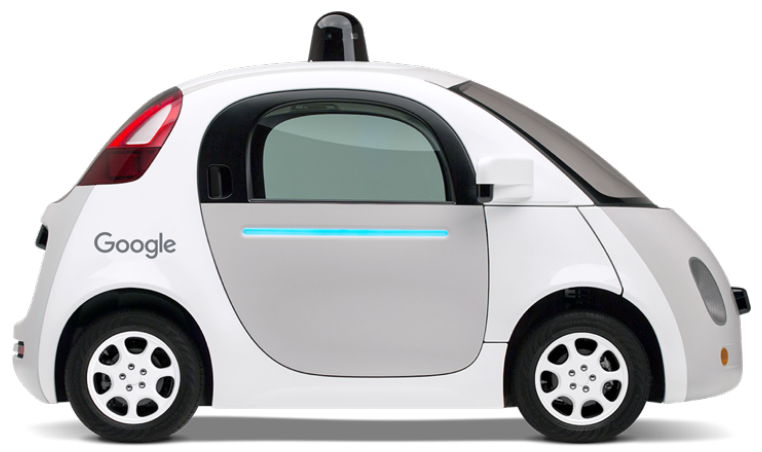

Figura 2. Firefly, o primeiro carro autônomo do Google.

Segundo Rodrigues (2018) o Brasil possui atualmente universidades trabalhando em protótipos de carros autônomos, tendo em foco o desenvolvimento do veículo em si, não em uma base de dados, até o momento da pesquisa. Baseado no que foi encontrado na literatura, até o presente momento, percebe-se a necessidade de uma base de dados para qualquer trabalho dessa complexidade. Atualmente não existe uma base de dados com placas de trânsito brasileiras, como existe, por exemplo, a base de dados belga da Visics (2009) ou a alemã da Stallkamp et al. (2011), que são utilizadas geralmente para testes e validações de reconhecimento de objetos, mas não possuem a compatibilidade para o nosso país, uma vez que as placas são diferentes.

Este trabalho aborda a criação e a validação de uma base de dados de imagens feitas para o reconhecimento de alguns objetos presentes no trânsito brasileiro, expondo os métodos de criação da base de dados e a forma de validação através do uso de DL, juntamente com testes feitos em cenários virtuais e reais.

Assim, este trabalho justifica-se pela necessidade de uma base de dados, para treinamento e testes, com elementos de trânsito mais comuns no Brasil para auxiliar futuros veículos autônomos que possam vir à trafegar no país.A forma de avaliação utilizada para a base de dados criada foi o desempenho da Yolo-v3, o estado da arte em detecção de objetos em tempo real (Redmon and Farhadi, 2018), ao ser treinada pela base.

\section{MÉTODOS}

Para este trabalho foi criada uma base de dados contendo os objetos a serem detectados, sendo esses objetos divididos nas seguintes classes: cones, pedestres, bicicletas, ciclistas, veículos, motocicletas, motociclistas, semáforos, 11 placas de regulamentação, 20 placas de advertência, 6 placas de serviços auxiliares, 1 placa de identificação e 1 placa de indicação, totalizando 47 classes. Cada classe representa um conjunto de objetos que deve ser tratado como equivalentes. Apesar de existirem diferentes tipos de carros, estes são considerados uma única classe, pois não se faz necessária a distinção entre os tipos ou cores dos carros para que um sistema posteriormente produza uma instrução de reação à presença do mesmo. Por outro lado cada placa de trânsito possui um significado único, sendo necessário que um sistema gere respostas individualizadas para cada instrução percebida nas placas.

Para assegurar o aprendizado de máquina em VC é preciso que a rede neural seja abastecida com imagens do mesmo objeto em diversas condições visuais (iluminação, posição do observador, obstrução por outros objetos) para a plena compreensão dos padrões que o identificam e os diferenciam de outros elementos.

Para criar a base foram utilizadas 1200 imagens de buscadores online e 512 imagens do Google Streetview que retratavam o trânsito brasileiro em condições realistas. Foram utilizadas 2000 imagens de carros retiradas de Krause et al. (2013) e 1300 imagens de Worcester (2018) contendo semáforos. Devido à insuficiência de imagens de placas de trânsito brasileiras em nas diversas condições disponíveis abertamente para a instrução da rede neural, adotou-se a opção de criação de elementos em softwares de modelagem gráfica para aumentar a base de imagens em circunstâncias visuais diferentes. Tal abordagem é similar ao utilizado 
por Pen (2018), que demonstrou as vantagens de utilizar softwares de modelagem gráfica no processo de criação de imagens para treinamento em sistemas de VC. Softwares de modelagem, como o Blender3D 2.79, permitem a obtenção de imagens realistas e altamente precisas, tornando-os um valioso recurso para treinamento e validação em VC. O Blender 3D permitiu a criação de placas diferentes, com diferentes iluminações e perspectivas em larga escala, como vistas na figura 3 .

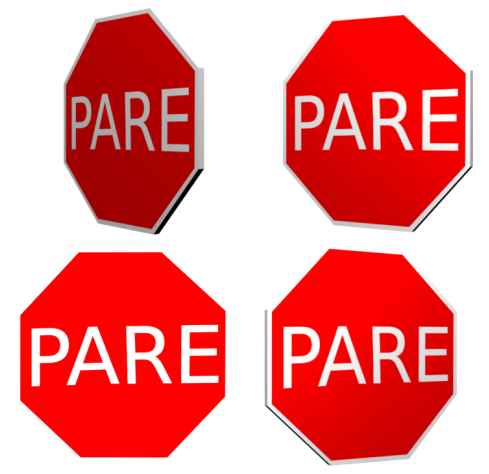

Figura 3. Imagens criadas no Blender 3D.

Após obter uma quantidade de, aproximadamente, 10000 imagens, foram aplicados filtros em todas essas imagens para que as mesmas tivessem variações para enriquecer o repertório como variação de brilho, contraste e temperatura da cor dentre outros, totalizando 79627 imagens disponíveis para posterior processamento.

Após terminar a aquisição de imagens, foi utilizado o software Yolo_mark AlexeyAB (2017) para a demarcação de objetos e rotulação em imagens em formato legível para o modelo sistema de detecção de objetos em tempo real Yolo-v3. Cada demarcação e rotulação exerce influência no treinamento, indicando o que a rede deve analisar para identificação de padrões. Toda a contraparte da demarcação se torna uma amostra negativa, que representa o que a rede neural deve ignorar, pois não faz parte do conjunto de objetos os quais deseja-se detectar. Quanto mais precisas, melhores as detecções; e quanto menos precisas, piores as detecções, podendo até mesmo não haver detecções por influência de informações inadequadas.

O modelo de rede neural convolucional ( $\mathrm{CNN}$ ) escolhida para este trabalho foi o Yolo-v3, que segundo Redmon and Farhadi (2018) é capaz de entregar alto desempenho em tempo real e com alta precisão. Para implementar a Yolo-v3 foi utilizado a API Darknet, que foi desenvolvida por Redmon and Farhadi (2018) para ser uma ferramenta otimizada na detecção de objetos em tempo real em conjunto com a Yolo-v3. O principal diferencial da Darknet em relação ao OpenCV e seu método baseado em Deep Neural Network (DNN) (biblioteca de DL do OpenCV), é sua compatibilidade com GPUs com Cuda Cores e Tensor Cores (NVIDIA, 2018a,c) visto que o uso de GPUs geram ganhos significativos de desempenho, enquanto o DNN da OpenCV se limita ao uso de CPUs. O OpenCV foi utilizado no apoio do desenvolvimento da base devido a suas bibliotecas que permitiam a visualização facilitada das saídas dos testes, no entanto não é uma biblioteca necessária para replicação dos resultados.
Foi feito o treinamento com a Yolo-v3, utilizando a extração do peso convolucional de um modelo pré-treinado, na resolução 608x608 pixels. A Yolo-v3 possui também um algoritmo de treino aleatório, que permite que diversas resoluções sejam treinadas em conjunto e 608x608 pixels foi o limite máximo estabelecido para um equilíbrio de taxa de frames e resolução para o hardware em questão.

A figura 4 contém o fluxograma simplificado da metodologia adotada durante a construção e validação da base.

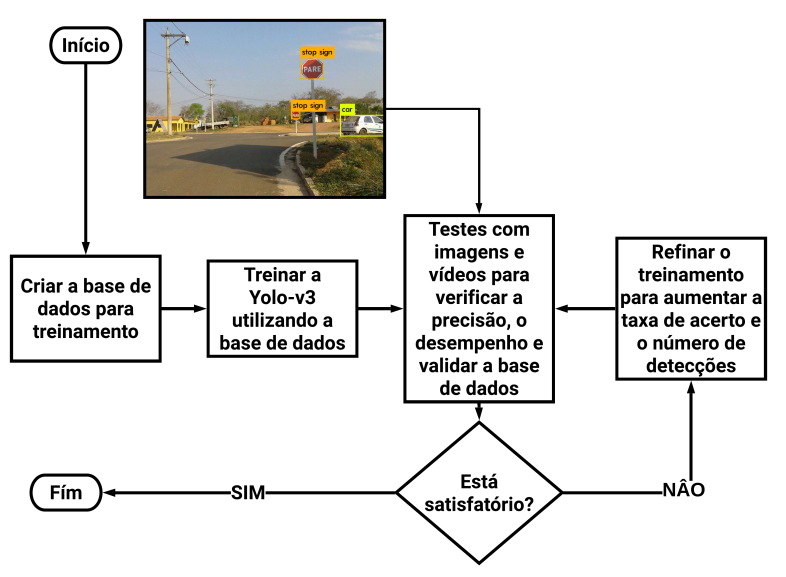

Figura 4. Fluxograma da metodologia do trabalho.

Durante o treinamento foi impedido que ocorresse a retropropagação para aumentar a velocidade do treinamento e executar um ajuste fino ao invés de transferir conhecimento. As âncoras, que são os pontos iniciais de varredura da rede de treinamento para identificar os objetos também foram recalculadas, inicialmente com 79627 imagens e treinado por 500000 iterações; a partir desse momento foi adicionado ao modelo treinado uma parte da base de dados para aplicações em VC da Microsoft, COCO versão 2017 (COCO, 2018). Com essa adição de 120000 imagens, totalizando 196876 imagens, a rede neural foi treinada do ponto em que estava até 901800 iterações, com um tempo total de 543,47h. Foram utilizadas para avaliação de desempenho do treinamento da Yolo-v3, o Mean Average Precision (mAP) e o Intersection over Union (IoU), que segundo Padilha (2018), são ferramentas para a avaliação da precisão de redes neurais convolucionais.

O processo de aferição do treinamento foi executado em cenários distintos onde houve a prévia contagem e rotulação de todos os elementos presentes. Para auxiliar no processo de validação do treinamento mais uma vez contou-se com a criação de cenários virtuais, a ferramenta de escolha foi a Unreal Engine 4 (UE4), que segundo Epic (2018), permite o treinamento de inteligência artificial de veículos autônomos com seus ambientes realistas. O fato da UE4 permitir o treinamento de IA's fez com que Shah et al. (2017) desenvolve-se o AirSim, uma plataforma cujo o objetivo é a pesquisa e o treinamento de Deep Learning, visão computacional e algoritmos de aprendizado reforçado para veículos autônomos, utilizando a UE4. Seguindo a mesma linha de pensamento, foram criados cenários virtuais para simular de forma controlada condições de circulação para 
teste da capacidade de detecção da rede neural, também utilizando a UE4.

O hardware utilizado foi um desktop com Processador Intel Core I5 6600K 4x @3.50 GHz, 32 GB de RAM DDR4 @2166 MHz, placa gráfica Nvidia RTX 2070 Gigabyte Gaming OC 8 GB GDDR6 e SSD Samsung EVO 860 250 GB. O sistema operacional foi Ubuntu 18.04 LTS com CUDA 10, cuDNN 7.4.2 e OpenCV 3.4.0.

\section{RESULTADOS}

Após o treinamento, o modelo obteve um mAP final de $65.60 \%$ e um IoU de $47.63 \%$, como visto na figura 5 .

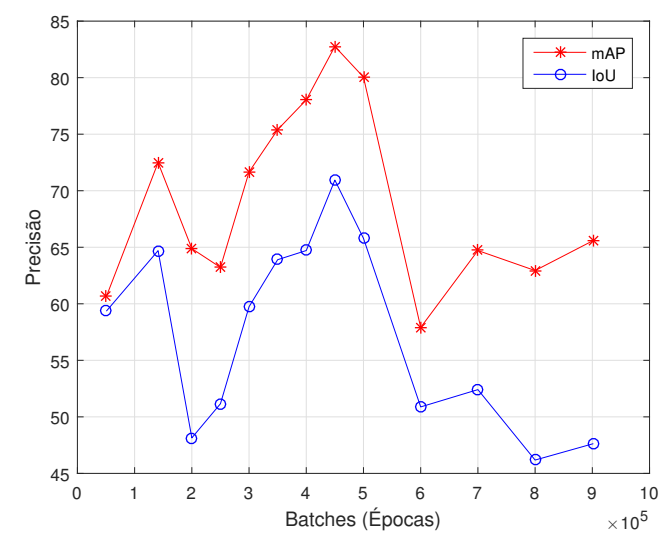

Figura 5. Precisão do mAP e IoU em relação às épocas.

A figura 6, contém exemplos de cenários virtuais que foram submetidas a testes, cujo resultados são apresentados na tabela 2 .

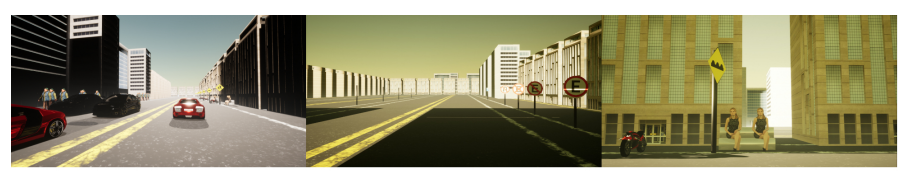

(a)

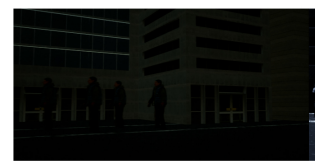

(d) (b)

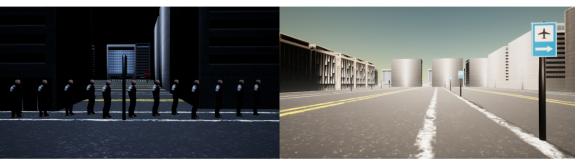

(e) (f)
Figura 6. Cenários virtuais. As características dos cenários são (a) há veículos, pedestres, placas e motocicletas, iluminação abundante; (b) há apenas placas de regulamentação e uma placa de serviço auxiliar, iluminação equivalente ao período da manhã; (c) há pedestres, motocicletas e placas de advertência, iluminação equivalente ao período da tarde; (d) há apenas pedestres, iluminação pobre; (e) há pedestres, uma placa de pare e uma placa de identificação, iluminação equivalente ao período noturno; (f) há apenas placas de serviços auxiliares, iluminação abundante.

A tabela 1 apresenta a quantidade total de objetos presentes em cada cenário virtual dos testes. Estes objetos de testes através da biblioteca Yolo-v3, são exibidos como resultados na tabela 2 no formato total de objetos classificados em relação ao total de objetos detectados e na mesma tabela entre parênteses constam a taxa de objetos detectados em relação ao total possível.

Quando um objeto é durante um teste é dito que o objeto foi "detectado"; quando além de detectado o objeto for corretamente alocado a sua categoria é dito é dito que ele foi "classificado".

Para testar a base em condições que simulasse o trânsito do veículo por ruas com diversas condições optou-se por criar vídeos (cinematics) utilizando a UE4. O vídeo foi processado pela Yolo-v3 como se fossem imagens captadas em tempo real. Na figura 6 é possível ver trechos dos diferentes cenários em que a foram realizados testes.

Na tabela 1 é possível identificar a quantidade de objetos presentes nos cenários, foi considerado a quantidade de objetos possíveis de serem detectados em cada quadro na contagem total de objetos do cenário.

Tabela 1. Quantidade de Objetos por Cenário.

\begin{tabular}{|c|c|}
\hline Cenários & Quantidade total de objetos \\
\hline $\mathrm{a}$ & 3247 \\
\hline $\mathrm{b}$ & 302 \\
\hline $\mathrm{c}$ & 900 \\
\hline $\mathrm{d}$ & 969 \\
\hline $\mathrm{e}$ & 2427 \\
\hline $\mathrm{f}$ & 290 \\
\hline
\end{tabular}

Na tabela 2 é possível identificar a quantidade de objetos detectados corretamente, ou seja, o objeto classificado com a classe correta. Desse montante estão excluídos os falsos negativos (quando um objeto pertencente a uma classe presente na base de dados não é identificado como tal) e inclusos os falsos positivos (quando objetos pertencentes a uma classe presente na base de dados é identificado equivocadamente como outro objeto). Na mesma tabela estão apresentados os valores de detecções máximas em cada cenário, permitindo identificar a taxa de sucesso.

Tabela 2. Quantidade de Acertos e Total de Detecções por Cenário.

\begin{tabular}{|c|c|c|c|c|c|}
\hline $\mathrm{C}$ & $\mathrm{M}$ & $\mathrm{T}$ & $\mathrm{N}$ & $\mathrm{MI}$ & $\mathrm{PI}$ \\
\hline \multirow{2}{*}{$\mathrm{a}$} & $737 / 737$ & $520 / 520$ & $328 / 332$ & $1458 / 1459$ & $186 / 188$ \\
& $(0,23)$ & $(0,16)$ & $(0,1)$ & $(0,44)$ & $(0,05)$ \\
\hline \multirow{2}{*}{$\mathrm{b}$} & $37 / 70$ & $64 / 65$ & $25 / 38$ & $72 / 81$ & $11 / 11$ \\
& $(0,12)$ & $(0,21)$ & $(0,08)$ & $(0,23)$ & $(0,04)$ \\
\hline \multirow{2}{*}{$\mathrm{c}$} & $72 / 73$ & $69 / 71$ & $44 / 45$ & $119 / 122$ & $56 / 56$ \\
& $(0,08)$ & $(0,08)$ & $(0,05)$ & $(0,13)$ & $(0,06)$ \\
\hline \multirow{2}{*}{$\mathrm{d}$} & $564 / 570$ & $620 / 631$ & $106 / 106$ & $615 / 617$ & $471 / 472$ \\
& $(0,58)$ & $(0,64)$ & $(0,11)$ & $(0,63)$ & $(0,49)$ \\
\hline \multirow{2}{*}{$\mathrm{e}$} & $1186 / 1186$ & $1160 / 1160$ & $679 / 679$ & $1010 / 1010$ & $791 / 791$ \\
& $(0,49)$ & $(0,48)$ & $(0,28)$ & $(0,42)$ & $(0,33)$ \\
\hline \multirow{2}{*}{$\mathrm{f}$} & $0 / 0$ & $0 / 0$ & $0 / 1$ & $1 / 1$ & $0 / 0$ \\
& $(0)$ & $(0)$ & $(0)$ & $(0)$ & $(0)$ \\
\hline
\end{tabular}

Condições do cenário: M - Manhã; T - Tarde; MI - Muita Iluminação; PI - Pouca Iluminação, C - Cenários. Entre parênteses a relação do total de detecções com a quantidade de objetos por cenário.

A figura 7, contém exemplos de cenários reais que foram submetidas a testes, cujo resultados são apresentados na tabela 3 . 
Nos cenários reais foram utilizadas imagens retiradas do Google Streetview, pois estas representam melhor o trânsito brasileiro. Importante salientar que, por restrições de software, o Google Street view permite captar imagens diurnas e não noturnas. Os cenários reais, utilizando somente imagens, são exibidos, na figura 7 .

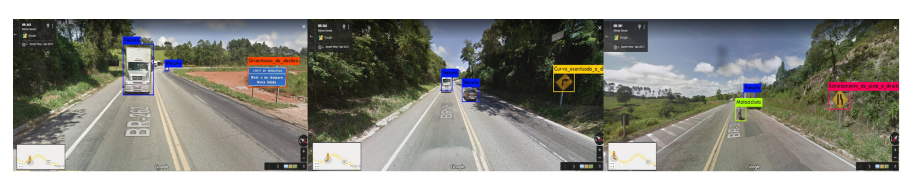

(a)

(b)

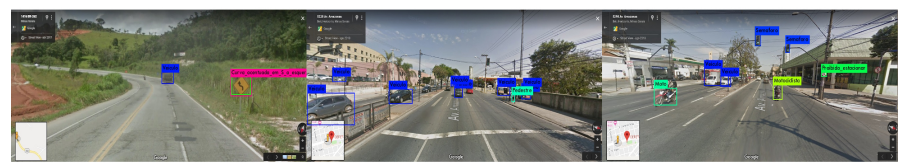

(d) (f)
Figura 7. Cenários reais. As características dos cenários são(a), três veículos e uma placa de identificação; (b), dois veículos e uma placa de advertência; (c), dois veículos, um motociclista e uma placa; (d)), dois veículos, um motociclista e uma placa; (e), há diversos veículos, um motociclista e semáforos; (f), há diversos veículos, um motociclista, semáforos e placas de regulamentação. .

Na tabela 3, é possível ver os resultados obtidos nos cenários reais, considerando a quantidade de objetos disponíveis, a quantidade de detecções realizadas, o percentual de detecções, o percentual de classificações e o tempo de detecção.

Tabela 3. Tabela demonstrando os resultados dos cenários reais.

\begin{tabular}{|l|c|c|c|c|c|}
\hline C $^{*}$ & TO & QD & PD & PC & T(ms) \\
\hline a & 4 & $3 / 4$ & $75 \%$ & $66,6 \%$ & 31 \\
\hline b & 3 & $3 / 3$ & $100 \%$ & $100 \%$ & 29 \\
\hline c & 4 & $3 / 4$ & $75 \%$ & $100 \%$ & 30 \\
\hline d & 4 & $2 / 4$ & $50 \%$ & $66,6 \%$ & 29 \\
\hline e & 30 & $9 / 30$ & $30 \%$ & $100 \%$ & 31 \\
\hline f & 17 & $7 / 17$ & $41 \%$ & $100 \%$ & 32 \\
\hline
\end{tabular}

C - Cenários; TO - Total de objetos; QD - Quantidade de detecções; PD - Percentual de deteç̧ões; PC - Percentual de classificações; T - Tempo total por imagem em milissegundos

\section{DISCUSSÕES}

Em relação ao ambiente virtual houve uma detecção de $21,5 \%$ de objetos, o qual $83 \%$ destes foram classificados corretamente. É possível perceber também na tabela 2 que a iluminação possui uma grande influência na capacidade de detecção da rede neural. Como demonstrado, cenários com melhor iluminação possuem mais objetos detectados, enquanto cenários com iluminação mais precária possuem piores resultados.

A condição de baixa luminosidade é também um desafio para a visão humana, o que é natural devido à perda de informação visual. Os resultados para essas condições representam uma oportunidade de destaque aos veículos autônomos por permitirem a incorporação de equipamentos de visão noturna, tornando a circulação em condições de baixa luminosidade mais segura em veículos autônomos do que em veículos convencionais.

Em relação ao ambiente real houve uma detecção de $61,8 \%$ de objetos, o qual $88 \%$ destes foram classificados corretamente. Em relação a rodovias, onde geralmente há um número menor de elementos a serem detectados por quadro, o Yolo-v3 consegue bons resultados.

Para uma comparação do desempenho da base com outros trabalhos, Martins (2017) faz uso de métodos do OpenCV para detecção de 5 placas de regulamentação brasileiras com uma rede neural perceptron de múltiplas camadas, utilizando 250 imagens para treino com um tempo total de $7,5 \mathrm{~h}$ de treinamento. O autor conseguiu atingir uma classificação de 96.6\% na classificação e um tempo de predição de $93 \mathrm{~ms}$ com imagens.

O trabalho de Sobrinho et al. (2016) faz uso de DL para a detecção de 19 placas de trânsito brasileiras, sendo 17 de regulamentação e 2 de advertência. Foram utilizadas 1300 imagens sintéticas, sendo 533 para treinamento, 267 para validação e 500 para testes. Seu modelo obteve uma classificação de $99.98 \%$, mas não foram testadas imagens de cenários reais.

Em comparação com trabalhos relacionados, este trabalho consegue uma precisão geral de $61,8 \%$ na classificação, com um conjunto maior de placas de trânsito com uma média de 30 fps.

O presente trabalho representa uma expansão do alcance das bases já desenvolvidas para o trânsito brasileiro, totalizando 39 classes. Valor bem acima das 19 classes da base desenvolvida por Sobrinho et al. (2016) e das 5 classes desenvolvidas por Martins (2017). O custo da expansão é a queda na taxa de precisão quando comparado aos outros trabalhos.

Suspeita-se que melhores resultados serão obtidos se ao invés de incorporar o modelo que estava sendo construído a um modelo já treinado (como foi feito nesse trabalho unificando parcialmente a COCO) se a base de imagens original for utilizada em conjunto com a base desenvolvida nesse trabalho para um treinamento único.

Foi possível explorar uma conjunção de outras tecnologias atuais para suprir restrições típicas da criação de uma base de dados, como a necessidade de grande volume de imagens para o treinamento eficiente. A solução foi a utilização de softwares de modelagem gráfica, que em seu estágio atual permitem a representação fidedigna de cenários reais.

A construção de cenários virtuais para treinamento de veículos autônomos tem sido adotada também por empresas (Epic, 2018) despontando como uma tendência para desenvolvimento econômico de novas tecnologias.

Todo o material produzido neste trabalho encontra-se disponível em: https://github.com/diegohaji/Base-de-dadosTransito-Brasileiro

\section{CONCLUSÃO}

Este trabalho criou uma base de dados e a treinou para validá-la, obtendo uma detecção de 21,5\% de objetos, o qual $83 \%$ destes foram classificados corretamente no cenário virtual; e uma uma detecção de $61,8 \%$ de objetos, 
o qual $88 \%$ destes foram classificados corretamente no cenário real.

A API Darknet consegue uma taxa média de 30 FPS com uma resolução interna da rede de 608x608, com o hardware utilizado, o que significa que hardwares melhores conseguem uma taxa de quadros maior, possibilitando até mesmo aumentar a resolução interna da rede, que aumenta a capacidade de detecções, mantendo o mesmo desempenho. Em relação a detecção em imagens, a API consegue um tempo médio de $30 \mathrm{~ms}$. Durante o treinamento e os testes foram constatadas algumas limitações:

- A biblioteca Yolo-v3 não consegue rastrear a movimentação dos objetos de um quadro a outro como faz o processamento de visão em seres humanos, impedindo que o cálculo de velocidade e a projeção de posição seja possível;

- Também em decorrência da detecção quadro a quadro sem rastreamento do objeto é que de um quadro para o outro o mesmo objeto pode deixar de ser detectado, principalmente quando a imagem apresenta elevada densidade de objetos;

- A geração e análise de relatórios não está implementada de forma suficiente nas ferramentas utilizadas, demandando ajustes próprios nas ferramentas capazes de analisar a massa de dados produzida.

A base de dados resultante deste trabalho representa um dos primeiros esforços de mapeamento da sinalização de trânsito brasileira para utilização em VC, algo essencial para viabilizar que veículos autônomos e similares possam trafegar nas vias. Neste contexto, trabalhos podem surgir como: expansões da base de dados e aprimoramentos para produzir resultados ainda melhores tanto em abrangência quanto na precisão da detecção de objetos.

Como proposta de trabalho futuro, tem-se o aperfeiçoamento da base e a implementação de capacidade de rastreamento de objetos entre quadros.

\section{REFERÊNCIAS}

AlexeyAB (2017). Yolo mark. URL https://github. com/AlexeyAB/Yolo_mark.

COCO (2018). Coco - common objects in context. URL http://cocodataset.org/\#download.

Epic (2018). Unreal engine 4 - ia. URL https : //tinyurl. com/y3jkxm3m.

Krause, J., Stark, M., Deng, J., and Fei-Fei, L. (2013). 3d object representations for fine-grained categorization. In 4th International IEEE Workshop on 3D Representation and Recognition (3dRR-13). Sydney, Australia.

Krizhevsky, A., Sutskever, I., and Hinton, G.E. (2012). Imagenet classification with deep convolutional neural networks. In Proceedings of the 25th International Conference on Neural Information Processing Systems - Volume 1, NIPS'12, 1097-1105. Curran Associates Inc., USA. URL http://dl.acm.org/citation.cfm? id $=2999134$. 2999257.

LeCun, Y., Bengio, Y., and Hinton, G.E. (2015). Deep learning. Nature, 521(7553), 436-444. doi:10. 1038/nature14539. URL https://doi.org/10.1038/ nature14539.

Martins, M.A. (2017). Identificação de placas de trânsito através da classificação de imagens usando redes neurais artificiais. URL https:// repositorio.ufu.br/bitstream/123456789/19551/ 1/IdentificacaoPlacasTransito.pdf.

NVIDIA (2018a). Cuda zone. URL https://developer. nvidia.com/cuda-zone.

NVIDIA (2018b). Fundamentals of deep learning for computer vision. URL https://courses.nvidia.com/ courses/course-v1:DLI+C-FX-01+V2/about.

NVIDIA (2018c). Nvidia cudnn. URL https:// developer.nvidia.com/cudnn.

Patterson, J. and Gibson, A. (2017). Deep Learning: A Practitioner's Approach. O'Reilly Media, Inc., 2nd edition.

Pen, M. (2018). Blender for computer vision machine learning. URL https:// blog.thepixelary.com/post/174286685782/

blender-for-computer-vision-machine-learning.

Redmon, J. and Farhadi, A. (2018). Yolov3: An incremental improvement. CoRR, abs/1804.02767. URL http://arxiv.org/abs/1804.02767.

Rodrigues, L. (2018). Carros autônomos já começam a virar realidade. URL https ://tinyurl. com/y34olr9y.

Shah, S., Dey, D., Lovett, C., and Kapoor, A. (2017). Airsim: High-fidelity visual and physical simulation for autonomous vehicles. In Field and Service Robotics. URL https://arxiv .org/abs/1705.05065.

Sobrinho, M.V.O., Menezes, M.C., Nascimento, C.G.M., and Junior, G.B. (2016). Reconhecimento de sinais de transito utilizando deep learning. URL http://sistemas.deinf .ufma.br/anaisjim/ artigos/2016/201622.pdf.

Stallkamp, J., Schlipsing, M., Salmen, J., and Igel, C. (2011). The German Traffic Sign Recognition Benchmark: A multi-class classification competition. In IEEE International Joint Conference on Neural Networks, 1453-1460.

Visics (2009). Traffic sign recognition. URL http://www . vision.ee.ethz.ch/ ${ }^{\text {timofter/traffic_signs/. }}$

Waymo (2019). Waymo journey. URL https://waymo. com/journey/.

Worcester, Polytechnic, I. (2018). Wpi datasets. URL http://computing.wpi.edu/dataset.html. 